\title{
Mechanism Study of Capacitance Method for Steam-injecting Dryness Measuring
}

\author{
Jian Han, Xue Lan He, Hai Wei Mou \\ College of Electronics Science, Northeast Petroleum University, Daqing 163318,China \\ hanjian@nepu.edu.cn, chongjing2046@126.com,mhwmzh@163.com
}

Keywords: Steam quality; In-line measurement; Dielectric constant; Capacitor

\begin{abstract}
Regarding the importance of wet saturated steam dryness measurement, at the same temperature and pressure the dielectric constant of water is much greater than that of steam. When the wet steam flows by the capacitive sensor, the sensor capacitance changes with the steam wetness. A new method that barrel capacitor is presented to provide real-time and in-line measurement of steam quality. According to the calculation results and the theory data calibration, thus the present method solves the problems associated with industrial steam boilers and steam injection wells dryness in-line measurement.
\end{abstract}

\section{Introduction}

Production of heavy crude oil present special challenges compared to light crude oil because heavy crude oil is very viscous and does not flow easily. Production of heavy oil is becoming very common and steam injection is widely used to reduce the viscosity of heavy crude oil. In order to extract oil more effectively, one must know the quality of steam, so the measurement of steam quality in the steam injection is important. However, exist methods need complicated and expensive devices but can not get the quality of steam in real time[1]. Some techniques are promoted to achieve real-time, on-line measurement, such as measuring the refractive index, X-ray or microwave attenuation etc. Those methods are complicated and some of them remain in research stage. This paper introduce a real time steam quality measurement system, this system calculate the steam quality with capacitance value that measured by sensor under certain temperature and pressure.

\section{Measurement principle}

The dielectric properties of water continue to be of considerable interest, both for pure water as well as for water mixed with and bound in other materials [2]. At $100^{\circ} \mathrm{C}$ the real part of the static relative permittivity of water changes from about 56 for the liquid phase to approximately 1.012 as a gas [3].The dielectric constant of water is much greater than that of steam at the same temperature and pressure, this large difference in permittivity between liquid water and steam provides the basis for measuring quality with a capacitive sensor. When the wet steam flows by the capacitance sensor, the sensor capacitance changes with the steam wetness. The relative capacitance change caused by the steam wetness change, regardless of the sensor geometry.

Fig. 1 shows the principle of capacitance method. Accordingly, steam value can be calculated by measuring the relative capacitance change.

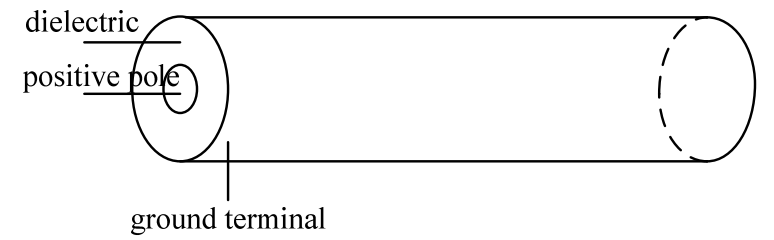

Fig.1 Schematic of the capacitance method

Steam quality is the parameter that quantifies the amount of condensed water present in steam. The formula for steam quality, denoted by $\mathrm{X}$ is: 


$$
\mathrm{X}=\frac{m_{g}}{m_{w}+m_{g}} \times 100 \% \text {. }
$$

Here, $m_{g}$ is the mass of the steam and $m_{w}$ is the mass of the liquid water[4].Quality is often expressed as a percentage.

For cylindrical structure of the capacitor its internal diameter of the capacitance is $R_{1}$ and outer diameter is $R_{2}$, if $\rho_{g}$ is the density of steam and $\rho_{w}$ is the density of liquid water and the volume percentage is $\mathrm{V}_{g}$ and $\mathrm{V}_{w}$, respectively. Here, $\varepsilon_{0}$ is vacuum permittivity. Capacitance value can be expressed as follows:

$$
\begin{aligned}
& C_{w}=2 \pi \varepsilon_{0} \varepsilon_{w} / \ln \frac{R_{2}}{R_{1}} . \\
& C_{g}=2 \pi \varepsilon_{0} \varepsilon_{g} / \ln \frac{R_{2}}{R_{1}} .
\end{aligned}
$$

According to Lichtenecker, equation may be written as:

$$
\varepsilon_{m}^{\frac{1}{2}}=\mathrm{V}_{\mathrm{w}} \varepsilon_{w}^{\frac{1}{2}}+\mathrm{V}_{\mathrm{g}} \varepsilon_{g}^{\frac{1}{2}} \text {. }
$$

where $\varepsilon_{m}$ is the permittivity value of the wet steam. $\varepsilon_{g}, \varepsilon_{w}$ are the permittivity values for pure steam and liquid water, respectively. $\rho_{w}, \rho_{g}, \varepsilon_{w}, \varepsilon_{g}$ can be known in the form of LAPWS-IF97at the certain temperature and pressure[5]. Refer with:Eq.1, Eq2, Eq.3, Eq.4,one can calculate the quality of the steam as following expression:

$$
\mathrm{X}=\frac{1}{\frac{\rho_{\mathrm{w}}}{\rho_{g}} \cdot \frac{\sqrt{\varepsilon_{m}}-\sqrt{\varepsilon_{g}}}{\sqrt{\varepsilon_{m}}-\sqrt{\varepsilon_{w}}}+1} .
$$

\section{Hardware and software design}

In order to ensure the data real-time, consistency and accurate calculation and analysis, so using computer collect and process data. Data acquisition and processing system is composed of hardware and software platform of two parts. Data acquisition includes temperature and pressure measurement and the dielectric constant of wet steam. Thermocouple temperature sensor is adopted to run normally in high - temperature environment. A pressure sensor which can reduce the sensor surface temperatures by cooling system is selected. Use barrel capacitor to measure dielectric constant so as to reduce effect of capacitor. The system is shown in Fig.2:
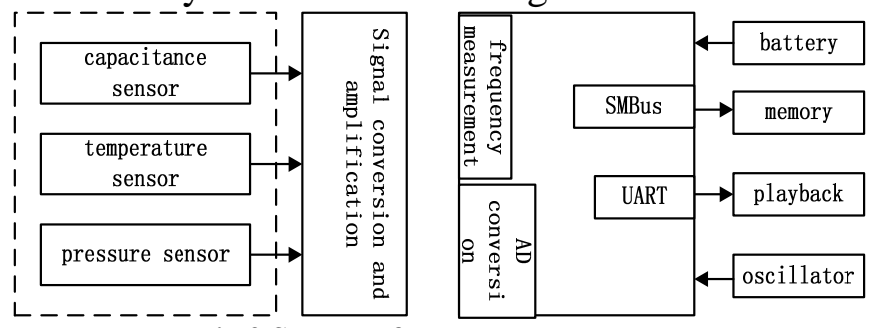

Fig.2 System of Hardware structure

Sensor Structure. Design the capacitance sensors is critical in the measuring process. Considered the wetness change is softer influence than electromagnetic environment and impure media disturbance. So it is necessary to design the sensor which must be meet field environment. A barrel capacitor which can be overcome above problem has been used to provide real-time and in-line measurement. The barrel capacitor have smaller edge effect and more practical.Fig.3 shows the structure of sensor. 


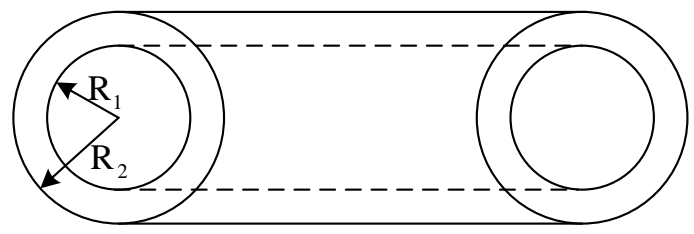

Fig.3 Structure of sensor

Software design. Multi-parameter measuring system completes the works by microcomputer in the wells. Test parameters must correspond to the system position underground. Sample value is measured and stored at regular intervals, so there is a one-to-one relationship between acquired parameters and time. Workers need to put down the system at a certain speed. Therefore, the relation between Well depth and test value is established. The system is built to a modular design, with composing of control module, measurement module, sending and receiving data module and Wireless Data Communication module etc.

\section{Data analysis}

It is a complex process to get the accurate steam value. Under certain temperature and pressure, one can know the parameters of water and steam by looking up the LAPWS-IF97 tables. Based on measured values, a multiple regression equation was generated by relevant software. The general equation is obtained in the range of permitted errors. Its calculate speed is faster than the formula provided by IAPWS-IF97.

Water dielectric constant:

$$
\varepsilon_{\mathrm{w}}=g_{w}(\mathrm{P}) h_{w}(t) \text {. }
$$

$g_{w}(\mathrm{P}), h_{w}(t)$ are given by the Taylor series expansion and kept some orders. The specific data in

IAPWS-IF97 are substituted in Eq.5, it forms a following matrix:

$$
\left[\begin{array}{c}
\varepsilon_{w 1} \\
\varepsilon_{w 2} \\
\varepsilon_{w 3} \\
\vdots
\end{array}\right]=\left[\begin{array}{cccc}
k_{11} & k_{12} & k_{13} & \cdots \\
k_{21} & k_{22} & k_{23} & \cdots \\
k_{31} & k_{32} & k_{33} & \cdots \\
\vdots & \vdots & \vdots
\end{array}\right]\left[\begin{array}{c}
x_{1} \\
x_{2} \\
x_{3} \\
\vdots
\end{array}\right] .
$$

Where, $\boldsymbol{\varepsilon}$ can be known by looking up IAPWS-IF97, $x$ is would be solved coefficients, then computation expression $\mathbf{X}=\left(\mathbf{K}^{T} \mathbf{K}\right)^{-1} \mathbf{K}^{T} \boldsymbol{\varepsilon}$ [6]. The result is shown in the table1.

Table1.the coefficients of equation of bivariate

\begin{tabular}{|c|c|c|c|}
\hline & $p^{2}$ & $p$ & 1 \\
\hline$t^{7}$ & $-5.0417 \times 10^{-19}$ & $1.3419 \times 10^{-17}$ & 0 \\
\hline$t^{6}$ & $4.8033 \times 10^{-16}$ & $-1.1172 \times 10^{-14}$ & 0 \\
\hline$t^{5}$ & $-1.7948 \times 10^{-13}$ & $3.5198 \times 10^{-12}$ & 0 \\
\hline$t^{4}$ & $3.3066 \times 10^{-11}$ & $-4.9076 \times 10^{-10}$ & 0 \\
\hline$t^{3}$ & $-3.1104 \times 10^{-9}$ & $2.2314 \times 10^{-8}$ & -1.1260 \\
\hline$t^{2}$ & $1.3712 \times 10^{-7}$ & $1.8796 \times 10^{-6}$ & 9.2089 \\
\hline$t$ & $-2.3690 \times 10^{-6}$ & $-2.4277 \times 10^{-4}$ & -0.4046 \\
\hline 1 & $-1.7830 \times 10^{-5}$ & 0.0421 & 87.9869 \\
\hline
\end{tabular}

Four simulation results are shown from Fig.4 toFig.7 at different temperature and pressure. 


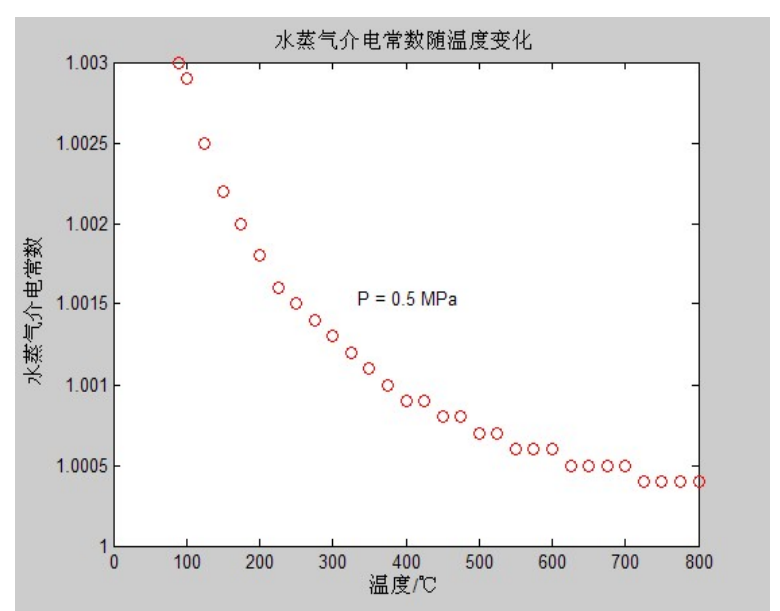

Fig. 4 Saturated steam dielectric constant changing with temperature

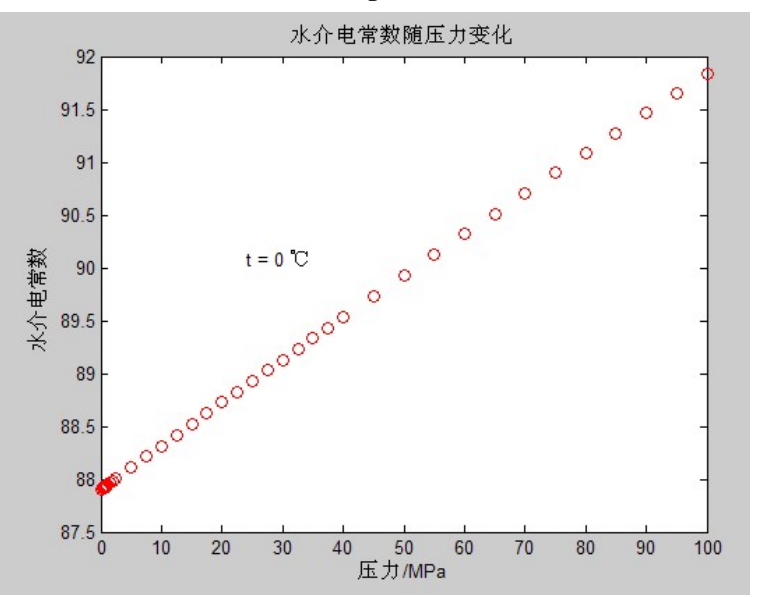

Fig. 6 Saturated water dielectric constant changing with pressure

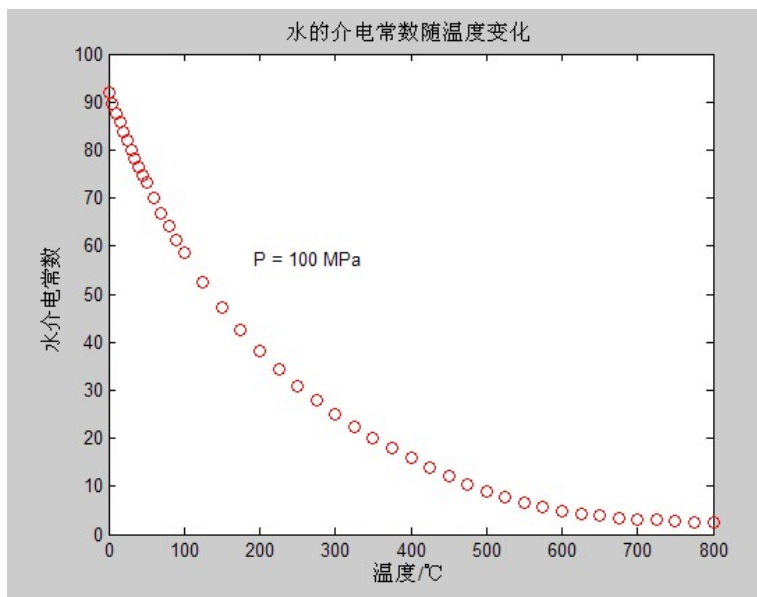

Fig. 5 Saturated water dielectric constant changing with temperature

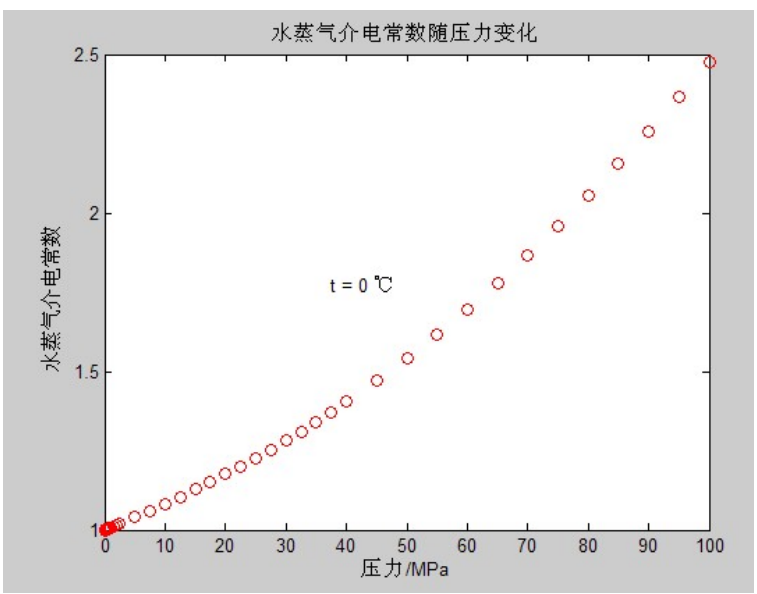

Fig.7 Saturated steam dielectric constant changing with pressure

\section{Summary}

Capacitance method is characterized by simple in measuring operation and data adequacy and reliable in the current methods and test system is simple with inexpensive and withstand hostile environment and without steam extraction sample. So it is worthy to research and develop steam in-line measurement. In addition, Steam contains impurity which is influence on dielectric constant bigger need to change process and methods for compensation.

\section{References}

[1] Steam and Water Sampling, Conditioning, and Analysis in the Power Cycle, ASME PTC 19.11-1997.NewYork,1997.

[2] Andrzej Kraszewski, editor, MICROWAVE AQUAMETRY Electromagnetic Wave Interaction with Water-Containing Materials, Piscataway, NJ, IEEE Press, 1996.

[3] NIST/ASME STEAM PROPERTIES DATA BASE: VERSION 2.21, NIST Standard Data Base 10, From International Association for the Properties of Water and Steam (IAPWS), 1995.

[4] Y. A. Cengel and M. A. Boles, Thermodynamics: An Engineering Approach, 5thP Edition, New York, 2006.

[5] ning de liang New capacitance sensor in the measurement of the steam humidity research and application Harbin Harbin engineering university.2007.

[6] basis of Mathematical statistical Tsinghua university press.1998. 